\title{
VLA RADIO CONTINUUM AND IRAS OBSERVATIONS OF THE RING NEBULAE AROUND WR 101 AND WR 113
}

\author{
C. E. CAPPA ${ }^{1}$ \\ Instituto Argentino de Radioastronomía, C.C. 5, 1894 Villa Elisa, Argentina; and Facultad de Ciencias Astronómicas y Geofísicas, \\ Universidad Nacional de La Plata, Paseo del Bosque s/n, 1900 La Plata, Argentina; \\ ccappa@fcaglp.fcaglp.unlp.edu.ar \\ W. M. Goss \\ National Radio Astronomy Observatory, P.O. Box O, Socorro, NM 87801; mgoss@aoc.nrao.edu
}

AND

S. Pineault

Département de Physique and Observatoire du Mont Mégantic, Université Laval, Ste-Foy, QC G1K 7P4, Canada; pineault@phy.ulaval.ca Received 2001 December 11; accepted 2002 March 15

\begin{abstract}
We report radio continuum observations at $1465 \mathrm{MHz}$ obtained with the Very Large Array (VLA) in the DnC configuration toward the ring nebulae associated with the stars WR 101 and WR 113, with resolutions of $\sim 38^{\prime \prime}$ and $30^{\prime \prime}$, respectively. IRAS images of the nebulae with resolutions of about $2^{\prime}\left(90 M_{\odot}, 40 \mathrm{~cm}^{-3}\right)$ are also analyzed. A remarkable resemblance among the optical, infrared, and radio images of these ring nebulae is observed. The VLA data indicate that Anon. WR 101 is thermal in nature. An ionized mass of $\approx 230 \pm 40 M_{\odot}$ and electron densities in the range $\approx 40-55 \mathrm{~cm}^{-3}$ were estimated for Anon. WR 101 . The derived ionized masses and electron densities in the inner and outer shells of the nebula related to WR 113 are $\approx 20 \pm 10 M_{\odot}, 180-500 \mathrm{~cm}^{-3}$ and $\approx 90 M_{\odot} 40 \mathrm{~cm}^{-3}$, respectively. Based on infrared data at 60 and $100 \mu \mathrm{m}$, the derived masses and temperatures for the dust component in the ring nebula around WR 101 are $0.3-1 M_{\odot}$ and $\approx 40 \mathrm{~K}$. The associated masses suggest that the ring nebula related to WR 101 and the outer arc associated with WR 113 consist of swept-up interstellar matter, while the relatively low ionized mass associated with the inner shell of the nebula around WR 113 may contain a nonnegligible contribution of expelled ejecta material. The derived electron densities for the nebula around WR 101 and the inner shell around WR 113 are comparable to electron densities for other W-R ring nebulae. Low filling factors are inferred for both nebulae. The nebulae probably originated during the current W-R phase of the stars.
\end{abstract}

Key words: ISM: bubbles — ISM: individual (Anon. WR 101, Anon WR 113) — stars: individual (WR 101, WR 113) — stars: Wolf-Rayet

\section{INTRODUCTION}

Wolf-Rayet (WR) stars are evolved massive stars that have lost a significant portion of their envelopes in the form of stellar winds. The mass lost by these stars, together with the interstellar matter swept up by the action of the stellar wind, is expected to appear as a circumstellar ring nebula. These nebulae are thermal in nature (e.g., Johnson \& Hogg 1965; Goss \& Lozinskaya 1995). Three different mechanisms are involved in their formation (Chu 1991). According to the dominant mechanism, they can be classified as (1) $R$ type, radiatively excited $\mathrm{H}$ II regions, (2) $E$ type, mainly consisting of stellar ejecta, and (3) $W$ type, stellar windblown bubbles. $R$-type nebulae are also further classified as shell-structured $\mathrm{H}$ II regions $\left(R_{S}\right)$ or amorphous $\mathrm{H}$ II regions $\left(R_{a}\right)$.

Ring nebulae are identified at optical (e.g., Chu, Treffers, \& Kwitter 1983; Miller \& Chu 1993; Marston, Chu, \& Garcia-Segura 1994a, Marston et al. 1994b), infrared (e.g., Marston 1991; Mathis et al. 1992), and radio wavelengths (e.g., Johnson \& Hogg 1965; Wendker et al. 1975; Goss \& Lozinskaya 1995; Cappa et al. 1999). Concentric rings originating in different phases of the evolution of the central star have

\footnotetext{
${ }^{1}$ Member of Carrera del Investigador Científico, CONICET, Argentina.
}

also been detected (Marston 1995a, 1995b). At present, about $25 \%$ of the 227 Galactic W-R stars (van der Hucht 2001) have associated circumstellar optical nebulae.

Only a few of these W-R nebulae have radio continuum studies performed with appropriate angular resolution, e.g., nebular diameter to resolution ratio $\geq 10-15$. Previous radio continuum observations of W-R ring nebulae obtained with high angular resolution were carried out using the Westerbork Synthesis Radio Telescope (WSRT), the Very Large Array (VLA), and the Synthesis Telescope of the Dominion Radio Astrophysical Observatory (DRAO). NGC 2359 and M1-M67 were observed with the WSRT telescope at 1.4 and $5 \mathrm{GHZ}$ with synthesized beams of $46^{\prime \prime}$ and 6" (Israel \& Felli 1976; Felli \& Perinotto 1979). The same telescope was used to observe NGC 6888 (around WR 136) at 0.6, 1.4, and $5 \mathrm{GHz}$ with synthesized beams less than $1^{\prime}$ (Wendker et al. 1975). G2.4-1.2 (around WR 102; Goss \& Lozinskaya 1995) and NGC 2359 (around WR 7; Cappa et al. 1999) were studied by using the VLA at $1.465 \mathrm{GHz}$, with angular resolutions of $19^{\prime \prime}$ and $30^{\prime \prime}$, respectively. The nebulae around WR 134 and WR 130 were analyzed using the DRAO synthesis telescope at $21 \mathrm{~cm}$ with angular resolution of $\approx 1^{\prime}$ (Pineault et al. 1993; Gervais \& St-Louis 1999; Cichowolski et al. 2001). There is good agreement between the optical and the radio features for most of these nebulae. Ionized masses of 60 and $70 M_{\odot}$ were derived for G2.4-1.2 and the 
windblown filamentary shell in NGC 2359, respectively, indicating that these nebulae mainly consist of swept-up interstellar matter. On the contrary, ionized masses in the range 1-6 $M_{\odot}$ were derived for M1-M67, NGC 6888, and the nebula linked to WR 134 (Wendker et al. 1975; Israel \& Felli 1976; Gervais \& St-Louis 1999). For the case of NGC 2359 , these radio observations helped in clarifying the origin of the nebula, since previous mass determinations had suggested lower masses and hence a possible E-type origin.

In this paper we describe radio continuum observations at $1465 \mathrm{MHz}$ toward the ring nebulae associated with WR 101 and WR 113 by using the VLA. The main goals are to investigate the nature of the sources and to perform detailed imaging. Radio continuum observations lead to estimates of electron densities and ionized masses, independent of extinction. Complementary high-resolution IRAS data were also obtained from IPAC to determine the properties of the dust.

The optical ring nebula associated with WR 101 was identified in $\mathrm{H} \alpha$ by Marston et al. (1994b). The nebula appears as a faint arc of diffuse emission with a major axis of $\approx 11^{\prime}$. The W-R star appears superposed on the eastern edge of the nebula (see their Fig. 12). The nebula is bright to the east, while the fainter emission to the west is poorly defined. According to these authors, the star has moved to its current position, illuminating that side of the nebula. The nebula was classified as W/E type (Marston 1997). Marston (1996) identified an infrared shell $80^{\prime}$ in diameter in the IRAS data, which was interpreted as a windblown bubble created by the previous O-type star phase.

WR 113 belongs to the eclipsing binary system CV Ser and is a probable member of the Ser OB2 association (e.g., van der Hucht 2001). González \& Rosado (1984) discovered a double optical structure around the star, which consists of an inner ring $4^{\prime}$ in radius and an outer arc $9^{\prime}$ in radius, located at the northern edge of the bright $\mathrm{H}$ II region Sh 54 (Sharpless 1959). They suggest that the inner ring originated by the action of the stellar wind of the W-R star on the surroundings. The outer ring could have been formed in the first stages of the $\mathrm{W}-\mathrm{R}$ phase or in a phase previous to the W-R stage. They suggest that the outer ring is photoionized by HD 168112 (an 5.5f-type star belonging to Ser OB2), while the characteristics of the optical emission of the inner ring are compatible with low-velocity shock emission. $\mathrm{H} \alpha$ images of the nebula (Miller \& Chu 1993; Esteban \& Rosado 1995) show the inner structure as an elongated ring- like feature, while the larger one has a semicircular shape (Gonzalez \& Rosado 1984). Based on detailed spectroscopy, Esteban \& Rosado (1995) conclude that photoionization by the W-R star is the main source of excitation, find no clear evidence for expansion, and suggest that the nebula is of type $R_{S}$. The observed line widths are larger close to the center of the nebula than near their borders, probably indicating a higher degree of internal turbulence at the center. This effect could have originated in the mechanical interaction of the wind of the central star or in the presence of a bubble expanding at a lower velocity (Esteban \& Rosado 1995).

Table 1 summarizes the parameters of the W-R stars WR 101 and WR 113. WR 101 and WR 113 are carbon-rich latetype stars. These kinds of objects have infrared excesses due to the presence of circumstellar dust shells (see Williams 1995). WR 113 has a persistent circumstellar dust shell (Williams, van der Hucht, \& The 1987), and temporal declines in brightness have been interpreted as occasional eclipses due to temporary condensing clouds formed in the wind-wind collision zone around the companion (Veen et al. 1998).

In the following sections we describe the VLA observations and infrared data $(\S 2)$. The radio and infrared results corresponding to the ring nebulae are analyzed and discussed in $\S 3$, while the main conclusions are summarized in $\S 4$. The two nebulae were detected in the NRAO VLA Sky Survey (Condon et al. 1998) but the quality of the images is low because of confusion arising from numerous sources in the galactic plane.

\section{DATA SETS}

\subsection{VLA Observations and Reduction}

The two fields were observed in the radio continuum at $1465 \mathrm{MHz}(20 \mathrm{~cm}$ ) by using the Very Large array (VLA) of the NRAO in the DnC configuration on 2000 July 3 and 5. The sources $1328+307\left(3 \mathrm{C} 286 ; \mathrm{S}_{1.46 \mathrm{GHz}}=14.9 \mathrm{Jy}\right)$ and $1748-253\left(\mathrm{~S}_{1.46 \mathrm{GHz}}=1.3 \mathrm{Jy}\right)$ were used as primary and secondary flux density calibrators, respectively. The bandwidth was $50 \mathrm{MHz}$ and the total integration time per source, about 2 hours. The main parameters of the observed fields are listed in Table 2.

The data were edited, calibrated, and imaged using Astronomical Image Processing System tasks. Data corresponding to WR 101 were also self-calibrated. The images were made using the VTESS task with total flux densities of 3.1

TABLE 1

Main Parameters of the W-R Stars and Related Ring Nebulae

\begin{tabular}{|c|c|c|c|c|c|c|}
\hline Star & $\begin{array}{l}\text { R.A., Decl. } \\
\text { (B1950) }\end{array}$ & Spectral Type ${ }^{\mathrm{a}}$ & $\begin{array}{c}A_{V} \\
(\mathrm{mag})\end{array}$ & $\begin{array}{c}d \\
(\mathrm{kpc})\end{array}$ & $\begin{array}{c}\dot{M} \\
\left(10^{-5} M_{\odot} \mathrm{yr}^{-1}\right)\end{array}$ & $V_{w}\left(\mathrm{~km} \mathrm{~s}^{-1}\right)$ \\
\hline WR 101 (DA 3) ............ & $\left(17^{\mathrm{h}} 41^{\mathrm{m}} 53^{\mathrm{s}} .9,-31^{\circ} 49^{\prime} 4^{\prime \prime}\right)$ & WC 8 & $7.34^{\mathrm{b}}, 7.71^{\mathrm{c}}$ & $3.2^{\mathrm{a}}, 6.9^{\mathrm{b}}$ & & $1600^{\mathrm{d}}$ \\
\hline WR 113 (HD 168206)... & $\left(18^{\mathrm{h}} 16^{\mathrm{m}} 19^{\mathrm{s}} 8,-11^{\circ} 39^{\prime} 16^{\prime \prime}\right)$ & $\mathrm{WC} 8 \mathrm{~d}+\mathrm{O} 8-\mathrm{O} \mathrm{IV}$ & $3.23 \pm 0.1^{\mathrm{a}}$ & $1.8^{\mathrm{a}}, 2.5,{ }^{\mathrm{b}} 2.0^{\mathrm{e}, \mathrm{f}}$ & $<5.6^{\mathrm{g}}, 2.0 \pm 0.3,{ }^{\mathrm{h}} 2.4^{\mathrm{f}}$ & $1400^{\mathrm{i}}, 1890^{\mathrm{j}}$ \\
\hline
\end{tabular}

a Van der Hucht 2001.

b Conti \& Vacca 1990.

c Smith, Shara, \& Moffat 1990.

d Eenens \& Williams 1994 (mean values).

e Esteban \& Rosado 1995.

${ }^{\mathrm{f}}$ Nugis \& Lamers 2000.

g Leitherer, Chapman, \& Koribalski 1997.

${ }^{\text {h }}$ Lamontagne et al. 1996.

${ }^{\text {i }}$ Koesterke \& Hamann 1995.

j Nugis, Crowther, \& Willis 1998. 
TABLE 2

OBSERVATIONAL PaRAMETERS

\begin{tabular}{|c|c|c|}
\hline Parameter & WR 101 & WR 113 \\
\hline Field center $(\mathrm{B} 1950)$........... & $\left(17^{\mathrm{h}} 41^{\mathrm{m}} 53^{\mathrm{s}},-31^{\circ} 49^{\prime} 50^{\prime \prime}\right)$ & $\left.18^{\mathrm{h}} 16^{\mathrm{m}} 10^{\mathrm{s}},-11^{\circ} 40^{\prime}\right)$ \\
\hline 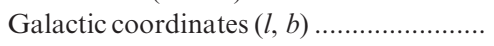 & $\left(357^{\circ} .47,-1.43\right)$ & $(18.91,+1.75)$ \\
\hline 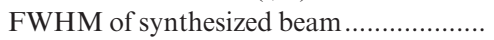 & $44 " .8 \times 32 ! .4$ & $39 " .8 \times 22 " .6$ \\
\hline 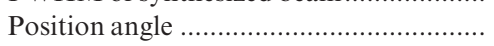 & $+49^{\circ}$ & $+82^{\circ}$ \\
\hline 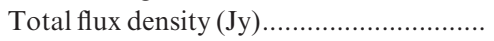 & $3.1 \pm 0.3$ & $11 \pm 1.0$ \\
\hline rms $\left(m J y\right.$ beam $\left.^{-1}\right)$ & 0.6 & 1.5 \\
\hline$S_{\nu}$ to $T_{B, 21}$ scale $\left[\mathrm{K}\left(\mathrm{mJy} \text { beam }{ }^{-1}\right)^{-1}\right] \ldots \ldots . .$. & 0.42 & 0.68 \\
\hline
\end{tabular}

and $11.0 \mathrm{Jy}$ for the fields corresponding to WR 101 and WR 113 , respectively, based on a conservative extrapolation to the center of the $u v$ plane.

\subsection{Infrared Data}

High-resolution (HIRES) IRAS images at 12, 25, 60, and $100 \mu \mathrm{m}$ of the ring nebulae were obtained from the Infrared Processing and Analysis Center (IPAC) ${ }^{2}$ and examined. The images have angular resolutions of $\simeq 0.7,0.8,1$. 3 , and $1{ }^{\prime} .8$ at $12,25,60$, and $100 \mu \mathrm{m}$, respectively.

\section{RING NEBULAE AROUND THE W-R STARS}

\subsection{Anon. WR 101 (G357.5-1.4)}

Figure 1 displays the radio continuum image at 1465 $\mathrm{MHz}$ as obtained with the VLA. The cross indicates the position of the star. At radio wavelengths, the nebula shows a complete and patchy ring structure about $12^{\prime}$ in diameter, centered at $\alpha=17^{\mathrm{h}} 41^{\mathrm{m}} 50^{\mathrm{s}}, \delta=-31^{\circ} 48^{\prime} 0$ (B1950). The source appears as a bright ring, with a faint emission connecting the southeast and the southwest. A strong radio

${ }^{2}$ IPAC is funded by NASA as part of the IRAS extended mission under contract to the Jet Propulsion Laboratory.

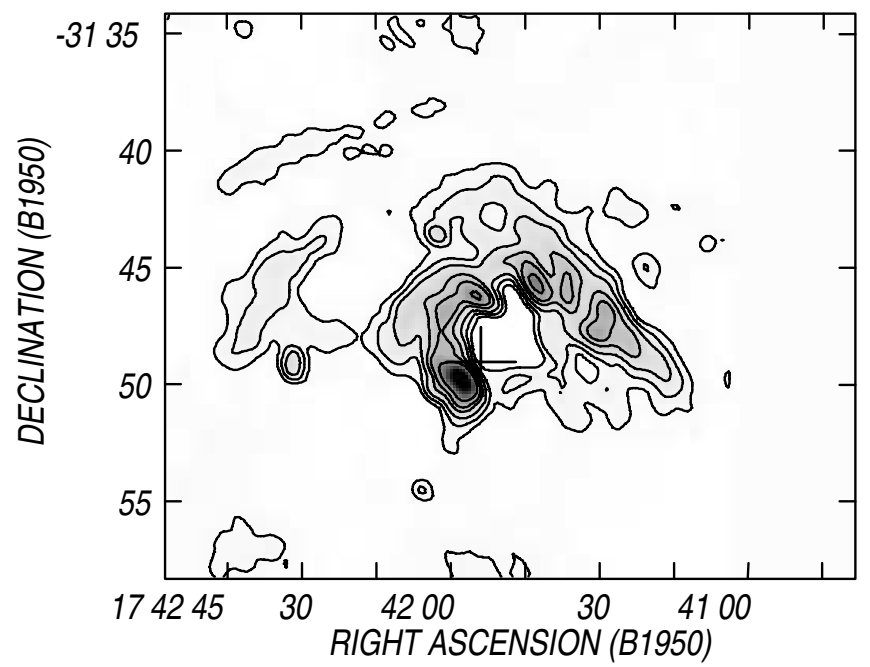

FIG. 1.-VLA image of the radio continuum emission of Anon. WR 101 at $1465 \mathrm{MHz}$ (synthesized beam $=44$ ". $8 \times 32$ " 4 , P. A. $=+49^{\circ}$ ). The gray scale corresponds to -0.1 to $120 \mathrm{mJy}^{\text {beam }}{ }^{-1}$. The contours are $2,5,10,20$, 30 , and $50 \mathrm{mJy}$ beam $^{-1}$. The cross indicates the position of WR 101 . source is detected in the southeastern section, close to the position of the $\mathrm{W}-\mathrm{R}$ star. The comparison between the radio and the $\mathrm{H} \alpha$ (Marston et al. 1994b) images shows good correlation in the northern, eastern, and western sections of the ring, while the weak radio emission connecting the southeastern and southwestern regions is not detectable in the $\mathrm{H} \alpha$ image.

Based on the VLA image, we derive a flux density $S_{1.46 \mathrm{GHz}}=3.1 \pm 0.3 \mathrm{Jy}$ for the structure. The source was detected by Haynes, Caswell, \& Simons (1978) in the 4.85 $\mathrm{GHz}$ survey, with a flux density $S_{4.85 \mathrm{GHz}}=2.8 \pm 0.3 \mathrm{Jy}$, suggesting a flat spectral index. The parameters of the H II region are listed in Table 3. The FWHM of the source is indicated in arcminutes and in parsecs. The emission measure EM, the rms electron density $n_{e}$, and the ionized mass $M_{i}$ were estimated from the expressions by Mezger \& Henderson (1967), assuming an electron temperature of $10^{4} \mathrm{~K}$, $10 \%$ abundance of singly ionized $\mathrm{He}$, and a distance of 3.2 kpc (van der Hucht 2001). The quoted distance error $(0.8$ $\mathrm{kpc}$ ) corresponds to a typical uncertainty of $\Delta M_{V}=0.5$ mag for the W-R star (e.g., van der Hucht 2001). The determination of the actual ionized mass and electron density relies on the geometry of the nebula. The derived values $n_{f}$ $\left(=n_{i} f^{-0.5}\right)$ and $M_{f}\left(=M_{i} f^{0.5}\right)$ are also listed in Table 3. Based on the uncertainty in distance, the error in the derived mass is $\approx 50 \%$. We estimated the volume-filling factor $f$ based on the radio and optical images. Assuming that the nebula is a section of a shell of about 1.0 in thickness with $25 \%-50 \%$ of its surface covered by ionized gas, $f \simeq 0.15-0.30$. Uncertainty in the filling factor is difficult to evaluate. However, since the ring appearance is well defined both at optical and radio wavelengths, the filling factor uncertainty relies on the fraction of the shell surface that is covered by ionized material. The original ambient density obtained by distributing

TABLE 3

Derived Physical Parameters of the Ring Nebulae

\begin{tabular}{|c|c|c|c|}
\hline \multirow[b]{2}{*}{ W-R STAR } & \multirow[b]{2}{*}{ WR 101} & \multicolumn{2}{|c|}{ WR 113} \\
\hline & & Inner & Outer \\
\hline$S_{1.4 \mathrm{GHz}}(\mathrm{Jy}) \ldots \ldots \ldots \ldots \ldots \ldots \ldots \ldots \ldots \ldots \ldots \ldots \ldots \ldots$ & $3.1 \pm 0.3$ & $4.1 \pm 0.4$ & $2.7 \pm 0.3$ \\
\hline Adopted distance $(\mathrm{kpc}) . . . . .$. & $3.2 \pm 0.8$ & $2.0 \pm 0.6$ & $2.0 \pm 0.6$ \\
\hline 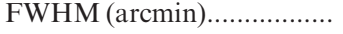 & $8.2 \times 8.3$ & $7.7 \times 2.8$ & $\approx 12 \times(\approx 3)$ \\
\hline Size $(\mathrm{pc})$ & $7.6 \times 7.7$ & $4.5 \times 1.6$ & $7 \times 2$ \\
\hline $\operatorname{EM}\left(10^{3} \mathrm{pc} \mathrm{cm}^{-6}\right) \ldots \ldots \ldots \ldots \ldots$ & 5.6 & 23.8 & 9.2 \\
\hline$n_{e}\left(\mathrm{~cm}^{-3}\right) \ldots \ldots \ldots \ldots \ldots \ldots \ldots \ldots \ldots \ldots \ldots \ldots \ldots$ & 21 & 70 & 40 \\
\hline$M_{i}\left(M_{\odot}\right) \ldots \ldots \ldots \ldots \ldots \ldots \ldots \ldots \ldots \ldots \ldots \ldots \ldots$ & 500 & 74 & 90 \\
\hline Filling factor, $f \ldots \ldots \ldots \ldots \ldots \ldots \ldots$ & $0.15-0.30$ & $0.02-0.15$ & 1 \\
\hline 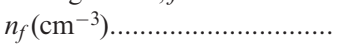 & $40-55$ & $180-500$ & $\ldots$ \\
\hline$M_{f}\left(M_{\odot}\right) \ldots \ldots \ldots \ldots \ldots \ldots \ldots \ldots \ldots \ldots \ldots \ldots \ldots \ldots \ldots$ & $190-265$ & $11-29$ & $\ldots$ \\
\hline
\end{tabular}


the actual ionized mass within the volume of a sphere of about $4.5 \mathrm{pc}$ in radius (a bit larger than the FWHM size) is $n_{0} \approx 25 \mathrm{~cm}^{-3}$, indicating that the nebula evolved in a relatively dense ambient medium.

The source centered at $\alpha=17^{\mathrm{h}} 41^{\mathrm{m}} 58^{\mathrm{s}} .0 \pm 0.1$, $\delta=-31^{\circ} 49^{\prime} 50^{\prime \prime} .2 \pm 0$. ."8 (B1950) has a flux density of $390 \pm 15 \mathrm{mJy}$ and a deconvolved size of $42^{\prime \prime}$. Although a chance superposition of a background source cannot be ruled out, the distribution of the ionized material suggests that this bright source is in fact related to the nebula. If its origin is thermal, the emission measure and electron density for the bright source are $\approx 10^{5} \mathrm{pc} \mathrm{cm}-6$ and $3 \times 10^{2} \mathrm{~cm}^{-3}$, respectively ( $f=1$ was assumed).

The ring nebula is clearly detected in the infrared at 25 , 60 , and $100 \mu \mathrm{m}$. Figure $2 a$ displays the $60 \mu \mathrm{m}$ HIRES image of the nebula, while Figure $2 b$ shows the superposition of the $60 \mu \mathrm{m}$ (gray scale) and radio continuum (contour lines) images. The comparison between the radio and the HIRES images shows excellent agreement between the IR and radio structures. The strong infrared emission to the southeast coincides with both the point source IRAS 17419-3150, which was detected in the four IRAS bands, and the smallscale structure IRAS X1741-318. The cataloged flux densities for the point source in the $12,25,60$, and $100 \mu \mathrm{m}$ bands
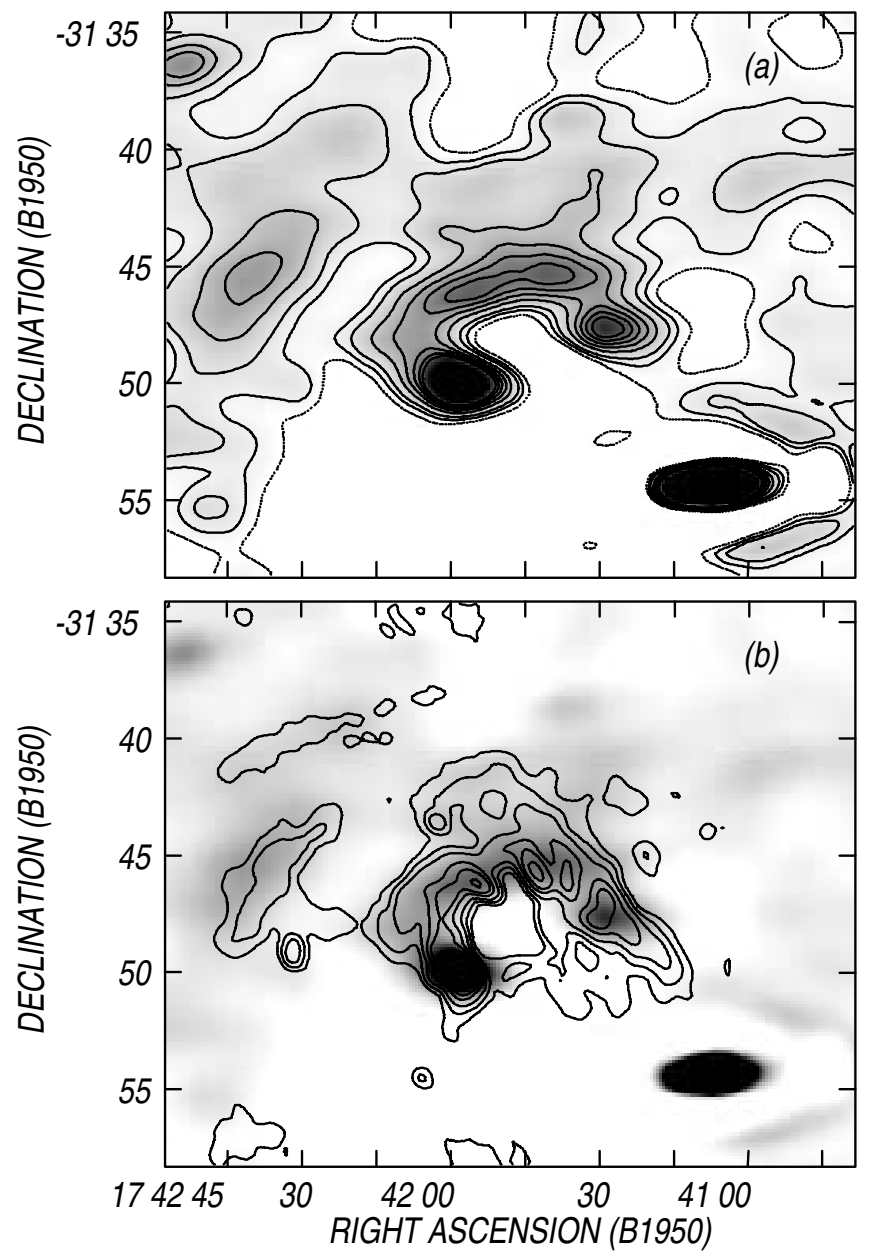

FIG. 2.-(a) HIRES infrared image at $60 \mu \mathrm{m}$ of Anon. WR 101 (G357.5-1.4), angular resolution $\simeq 1 ! 6 \times 1$ ' 0 . The gray scale corresponds to -10 to $170 \mathrm{MJy} \mathrm{sr}^{-1}$. The contours are $-10,0,10$, and 30 to 110 in steps of $20 \mathrm{MJy} \mathrm{sr}^{-1}$. (b) Radio continuum contours superposed on the infrared image of the nebula. are $4,11,95$, and $95 \mathrm{Jy}$, respectively. The colors of the source are compatible with an $\mathrm{H}$ II region (see Fig. 1 by Osterloh, Henning, \& Laundhardt 1997).

IR flux density determinations for extended structures depend on both the actual extent of the source and the amount of foreground and background emission. To quantify the associated uncertainties, flux densities were independently determined in two ways. First, these were obtained by simply integrating the original images over the area of the ring nebula and subtracting a fixed background corresponding to the lower contour line defining the nebula. The second method consisted in first applying a background filtering technique (Kothes \& Kerton 2002) to remove largescale features (different filter sizes were used and a filter size of $30^{\prime}$ found to yield the best results) and then simply using the DRAO IMVIEW task to integrate over the ring nebula (a twisted background fitted to the sides of the defining polygon is automatically subtracted). Both methods, applied to different polygons, gave similar results and these were averaged to give the final values and corresponding errors.

The final IR flux densities for the whole nebula are $315 \pm 40$ and $280 \pm 70 \mathrm{Jy}$ at 60 and $100 \mu \mathrm{m}$, respectively. The quoted errors are compatible with uncertainties of $30 \%$ and $70 \%$, for 60 and $100 \mu \mathrm{m}$ respectively, quoted by Fich \& Terebey (1996) for extended sources brighter than $100 \mathrm{Jy}$. The nebula is barely detectable at $12 \mu \mathrm{m}$. The measured flux densities indicate that the HIRES spectral energy distribution peaks near $60 \mu \mathrm{m}$ as was also found for NGC 6888 (around WR 136) and RCW 58 (around WR 40) (Mathis et al. 1992).

Using the flux densities at 60 and $100 \mu \mathrm{m}$, standard dust parameters (see Draine \& Lee 1984), and the expressions by Cichowolski et al. (2001), the derived dust temperature lies in the range $40-44 \mathrm{~K}$ for a spectral index of the dust absorption efficiency $n=1.5-2.0$. The estimated dust mass is $\approx 0.3-1.0 M_{\odot}$. The dust temperature is similar to the temperatures derived for bow shocks (Van Buren \& McCray 1988). The amount of dust and ionized masses suggests a gas-todust mass ratio of more than 200. Errors in dust mass estimates and, particularly, an overestimated ionized mass may explain this high value. Thus, the present gas-to-dust ratio favors lower filling factors than those adopted. We note also that only the ionized gas was taken into account since the amount of associated neutral and molecular material is unknown.

For $\mathrm{H}$ II regions, a strong correlation exists between the brightness temperature at $11 \mathrm{~cm}, T_{B, 11}$, and $I_{60}$, its equivalent at $60 \mu \mathrm{m}$ (e.g., Broadbent, Haslam, \& Osborne 1989; Fürst, Reich, \& Sofue 1987; Moon \& Koo 1994). In particular Broadbent et al. (1989) obtained the relation $T_{B, 11}=(6.4 \pm 1.7) \times 10^{-3} I_{60}$, where $T_{B, 11}$ is in kelvins and $I_{60}$ in megajanskys per steradian. Assuming a spectral index $\alpha=-0.1$, this relation becomes $T_{B, 21}=(23.0 \pm 6.1) \times$ $10^{-3} I_{60}$, or equivalently $I_{60}=(43.5 \pm 11.5) \times T_{B, 21}$, while for a typical nonthermal spectral index $\alpha=-0.5, I_{60}=$ $(34.0 \pm 9.0) \times T_{B, 21}$.

To test this relation, we convolved both the $60 \mu \mathrm{m}$ and VLA images to a common resolution of $1 ! 7$, blanking out an area centered on the bright source IRAS 17419-3150 to avoid inducing an excessive bias caused by the few bright pixels from this source. The plot in Figure $3 a$, corresponding to the ringlike structure near WR 101, shows the correlation between $I_{60}$ and $T_{B, 21}$. The slope fitted to the plot is $8.1 \pm 0.9$. This result is inconclusive. 

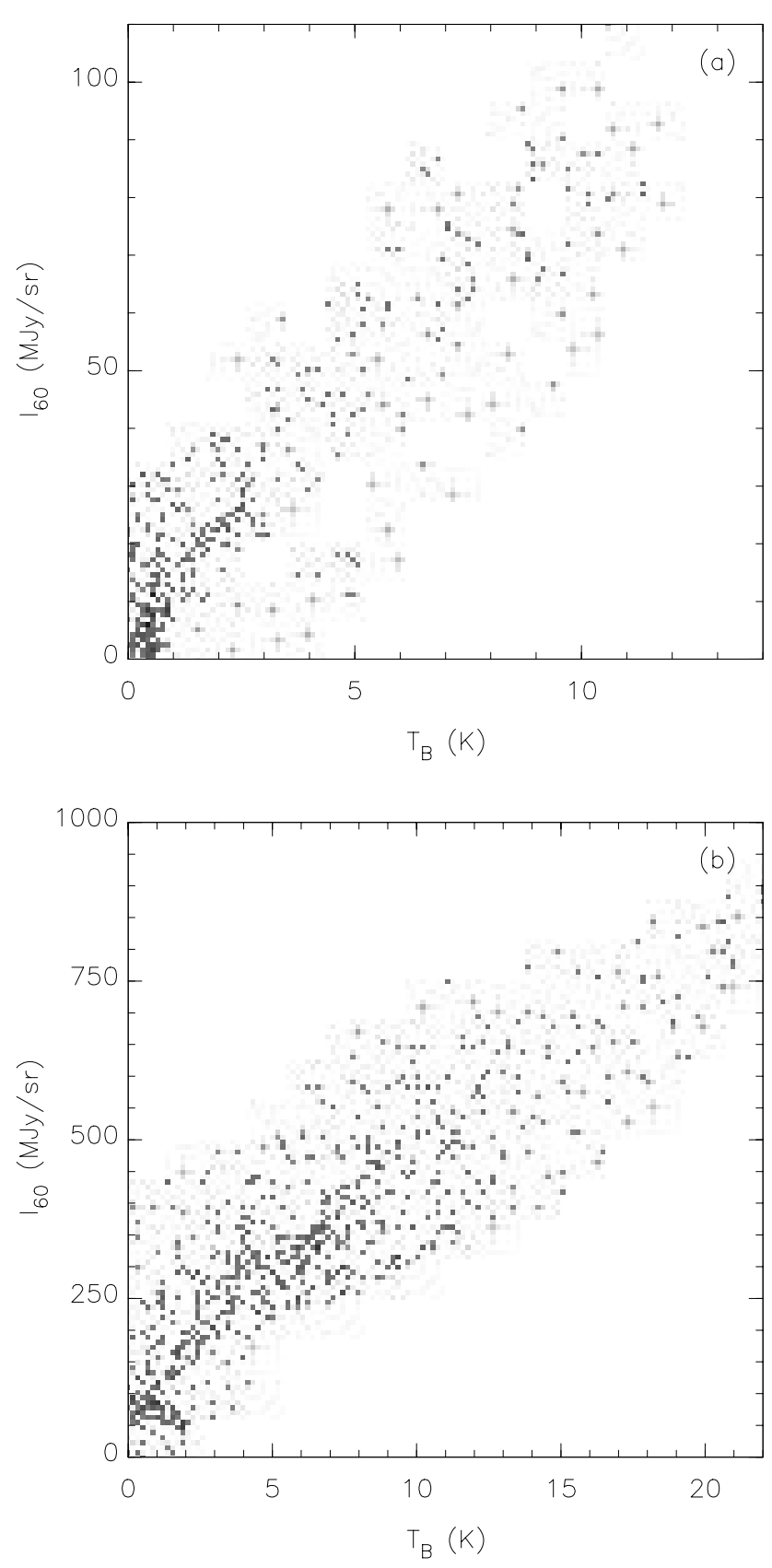

FIG. 3.-Plots of the $60 \mu \mathrm{m}$ brightness $I_{60}$ vs. the brightness temperature $T_{B}$ at $21 \mathrm{~cm}$, corresponding to (a) G357.5-1.4 (Anon. WR 101) and (b) G18.8+1.8. Note the different scales.

We carried out an independent check of this radio-infrared correlation by calculating the ratio $R$ of the brightness at $60 \mu \mathrm{m}$ expressed in janskys per beam area of the corresponding VLA image and the radio brightness in janskys per beam area. Fürst et al. (1987) have shown that $R<10$ for known supernova remnants and that $R$ varies between 500 and 1500 for $\mathrm{H}$ II regions. The value obtained for the region corresponding to Figure $3 a$ is $120 \pm 14$, intermediate between the thermal and nonthermal cases. Both this value and the $I_{60}$ versus $T_{B, 21}$ plot may suggest some contamination of nonthermal radio emission and /or large background uncertainties in the infrared image. Future multifrequency radio imaging is required to answer this question.
The point source IRAS 17411-3154 $\left(\alpha=17^{\mathrm{h}} 41^{\mathrm{m}} 7^{\prime \prime}\right.$, $\delta=-31^{\circ} 54 ! 4$ [B1950]), which does not have a radio counterpart, appears to be unconnected with the nebula.

On a larger scale, the IR ring nebula associated with WR 101 appears projected onto the northern section of the large IRAS shell (80' in diameter) found by Marston (1996) in the direction of the W-R star. Figure 4 shows the HIRES image at $60 \mu$, where the shell appears better defined than in the original IRAS data (see Fig. 1 by Marston 1996). By defining an area including both the small and the large IR features, we estimate the flux densities at 60 and $100 \mu \mathrm{m}$ as $(2.9 \pm 0.6) \times 10^{3}$ and $(7.5 \pm 1.5) \times 10^{3} \mathrm{Jy}$, respectively. The derived dust temperature is $\leq 30 \mathrm{~K}$, significantly cooler than that in the ring nebula itself. The dust mass in the large IR feature is $18 M_{\odot}$ (for $n=1.5$ ). Note that the diffuse radio emission north of the ring nebula (Fig. 1) is probably related to the large IR shell (Fig. 4).

The eccentric position of WR 101 (Fig. 1), close to the densest part of the nebula, could arise from the inhomogeneous structure of the interstellar medium (ISM) near the W-R star or from the motion of the star inside the shell. In the first case, the expansion of the $\mathrm{H}$ in region may have been favored to the north, south, and west of the W-R star because of the lower densities. The low radio emission from the southern part of the nebula may be suggestive of a blowout into the large IR shell. For the second explanation, the tangential motion of the star cannot be evaluated from the available proper-motion information $\left(\mu_{\delta} \cos \delta=-0.3 \pm 2.8\right.$ mas, $\mu_{\delta}=-0.4 \pm 2.8$ mas, from the Tycho-2 Catalogue; Hog et al. 2000) because of the large uncertainties.

An estimate of the dynamical age $t_{d}$ of the nebula can be obtained assuming that the nebula is in the energy-conserving phase (Weaver et al. 1977). The dynamical age in units of $10^{6} \mathrm{yr}$ is $t_{6}=\left(R_{s} / 27\right)^{1.67}\left(L_{36} / n_{0}\right)^{0.33}$, where $R_{S}$ is the radius of the nebula, $L_{36}$ the stellar wind luminosity in units of $10^{36} \mathrm{ergs} \mathrm{s}^{-1}$, and $n_{0}$ the original ambient density. Assuming conservative values for the mass-loss rate and the terminal velocity $\left(\dot{M}=1 \times 10^{-5} M_{\odot} \mathrm{yr}^{-1}\right.$ and $V_{w}=1500 \mathrm{~km}$ $\mathrm{s}^{-1}$, compatible with the values listed in Table 1), $L_{36}=7.2$. Adopting $R_{s}=3.9 \mathrm{pc}$ and $n_{0}=25 \mathrm{~cm}^{-3}, t_{d}=6 \times 10^{4} \mathrm{yr}$.

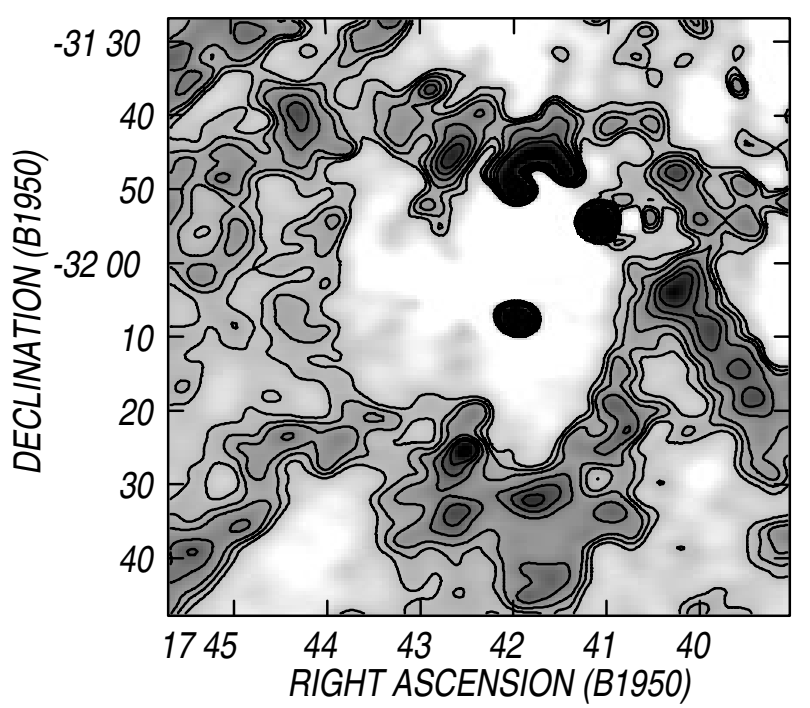

FIG. 4.-HIRES infrared image of the large IRAS shell at $60 \mu \mathrm{m}$ (angular resolution $=2 ! 2$ ). The gray scale corresponds to -20 to $60 \mathrm{MJy} \mathrm{sr}^{-1}$. The contours are $0,5,10,20,30$, and $40 \mathrm{MJy} \mathrm{sr}^{-1}$. 
This value is a lower limit since most of the ring nebulae seem to be in an intermediate stage between the energy- and the momentum-conserving cases (e.g., Chu et al. 1983). An upper limit to the expansion velocity $V_{\exp }$ can be derived following Weaver et al. (1977) as $V_{\text {exp }}=0.6 R_{s} / t_{6}=40 \mathrm{~km} \mathrm{~s}^{-1}$.

The estimated dynamical age suggests that the nebula probably originated during the W-R phase of the star. The upper limit to the expansion velocity is compatible with the observed expansion velocities in the range $15-40 \mathrm{~km} \mathrm{~s}^{-1}$ for most of the W-R ring nebulae classified as $W$ and $R$. A classification as $W$ - or $R$-type for G357.5-1.4 is compatible with the large ionized mass in the nebula.

\subsection{Anon. WR $113($ G18.8+1.8)}

The $1465 \mathrm{MHz}$ radio continuum emission distribution in the region of WR 113 is shown in Figure 5. As in Figure 1, the cross indicates the position of the W-R star. The radio image shows an extended source centered near $\alpha=18^{\mathrm{h}} 16^{\mathrm{m}} 7^{\mathrm{s}}, \delta=-11^{\circ} 41 ! 2$ (B1950), with the star close to its northeastern border. The brighter central radio emission region $(7 ! 7 \times 2 ! 8$ in diameter $)$ appears surrounded by a diffuse and fainter region $\left(17^{\prime} \times 13^{\prime}\right.$ in diameter $)$.

The strong radio source centered at $\alpha=18^{\mathrm{h}} 15^{\mathrm{m}} 05^{\mathrm{s}}$, $\delta=-11^{\circ} 46.5$ (B1950) $(\mathrm{G} 18.7+2.0$; Goss \& Day 1970) is a small $\mathrm{H}$ II region related to $\mathrm{Sh} 54$ and the open cluster NGC 6604, unconnected to the ring nebula. Lockman (1989) detected a radio recombination line from this source with an LSR velocity of $\approx 27 \mathrm{~km} \mathrm{~s}^{-1}$.

The comparison between the radio and the optical (see Fig. 1 by Miller \& Chu 1993; Esteban \& Rosado 1995) images shows that the extended radio source projected close to the star is the radio counterpart of the optical inner ring associated with the star. The western and southern sections of the ring nebula are very prominent at $1465 \mathrm{MHz}$, while the northeastern section is barely detectable. The radio emission near $\alpha=18^{\mathrm{h}} 16^{\mathrm{m}} 15^{\mathrm{s}}, \delta=-11^{\circ} 42$ ! 0 (B1950) and $\alpha=18^{\mathrm{h}} 16^{\mathrm{m}} 05^{\mathrm{s}}, \delta=-11^{\circ} 39 ! 3$ (B1950) correlates with a

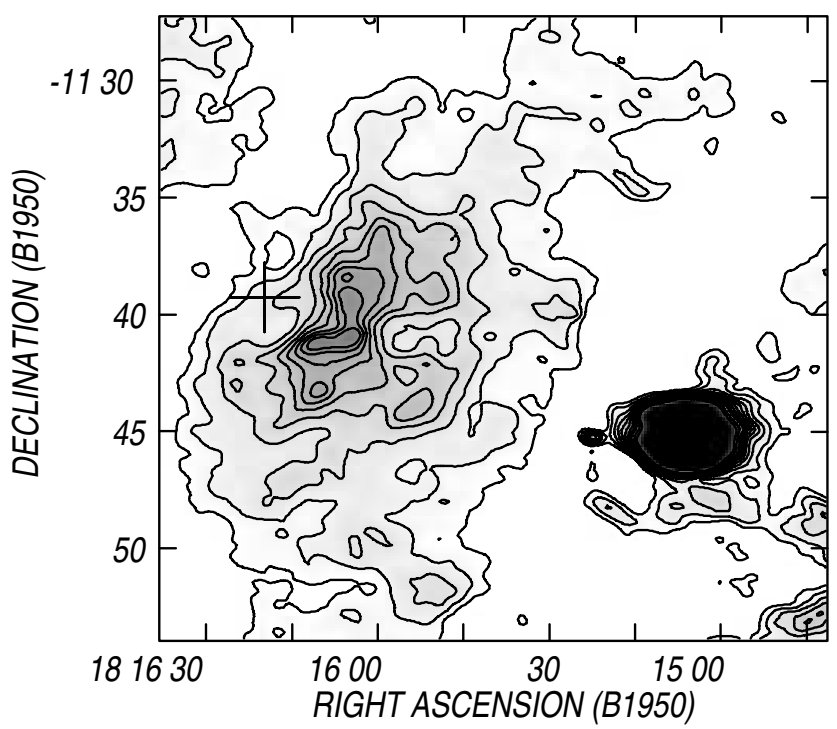

FIG. 5.-VLA image of the radio continuum emission of the nebula related to WR $113(\mathrm{G} 18.8+1.8)$ at $1465 \mathrm{MHz}$ (synthesized beam $=39^{\prime \prime} .8 \times 22^{\prime \prime} 6$, P.A. $=+82^{\circ}$ ). The gray scale corresponds to 1 to 90 mJy beam ${ }^{-1}$. The contours are $1,5,10,15,20,25,30,35,40,50$, and $60 \mathrm{mJy}$ beam $^{-1}$. The cross indicates the position of WR 113 . bright filament detected in $\mathrm{H} \alpha,[\mathrm{N}$ II], and [S II] (Miller \& Chu 1993; Esteban \& Rosado 1995). The region of low $\mathrm{H} \alpha$ and $[\mathrm{N}$ II] emission near the center of the optical ring also appears as a region of low emission at $1465 \mathrm{MHz}$ at $\alpha=18^{\mathrm{h}} 16^{\mathrm{m}} 18^{\mathrm{s}}, \delta=-11^{\circ} 40.5$ (B1950), suggesting that the ionized gas shows a minimum in this region. The strongest radio emission region near $\alpha=18^{\mathrm{h}} 16^{\mathrm{m}} 05^{\mathrm{s}}, \delta=-11^{\circ} 41^{\prime} 0$ (B1950), coincides with a region of faint optical emission, indicating that the dust lanes observed in the optical image obscure this portion of the nebula.

The faint extended radio emission detected to the west above $1 \mathrm{mJy}^{\text {beam }}{ }^{-1}$ (from $\alpha=18^{\mathrm{h}} 15^{\mathrm{m}} 40^{\mathrm{s}}, \delta=-11^{\circ} 35^{\prime}$ to $\alpha=18^{\mathrm{h}} 16^{\mathrm{m}} 15^{\mathrm{s}}, \delta=-11^{\circ} 50^{\prime}$ [B1950]) is associated with the outer optical ring identified by González \& Rosado (1984).

The infrared image of the nebula at $60 \mu \mathrm{m}$ is displayed in Figure 6 (gray scale). The infrared and radio images coincide. At infrared and radio wavelengths (see Reich et al. 1990), the nebula appears projected onto extended background emission probably related to the whole Sh 54 complex.

The radio counterpart of the optical nebula can be identified with the radio source $\mathrm{G} 18.8+1.8$, belonging to the $\mathrm{H}$ II region Sh 54 (RCW 167, W35). G18.8+1.8 was detected at $0.4 \mathrm{GHz}$ (Shaver \& Goss 1970a), $2.7 \mathrm{GHz}$ (Goss \& Day 1970; Reich et al. 1990), and 5 GHz (Goss \& Shaver 1970) with angular resolutions of $3^{\prime}, 8^{\prime}$ and $4 ! 3$, and $4 ! 1$, respectively. The flux density of the source was estimated to be $S_{0.4 \mathrm{GHz}}=11.5 \mathrm{Jy}\left(\approx 8^{\prime}\right.$ in size $)$ and $S_{5 \mathrm{GHz}}=20.1 \mathrm{Jy}\left(\approx 12^{\prime}\right.$ in size; Shaver \& Goss 1970b). These last authors conclude that the source is thermal in nature.

The main physical parameters derived from the present VLA data for both the inner and outer rings are summarized in Table 3. Our estimates are based on the same assumptions as for the nebula around WR $101\left(T_{e}=10^{4} \mathrm{~K}\right.$, $10 \%$ single ionized $\mathrm{He}$ abundance). The adopted distance is $2.0 \pm 0.6 \mathrm{kpc}$, in close agreement with the stellar distance (see Table 1). Based on $\left[\mathrm{S}_{\mathrm{II}}\right]$ line intensity ratios, Esteban \& Rosado (1995) derived $n_{[\mathrm{S} \mathrm{II}]} \simeq 1000 \mathrm{~cm}^{-3}$, indicating a highly inhomogeneous nebula. Since electron density esti-

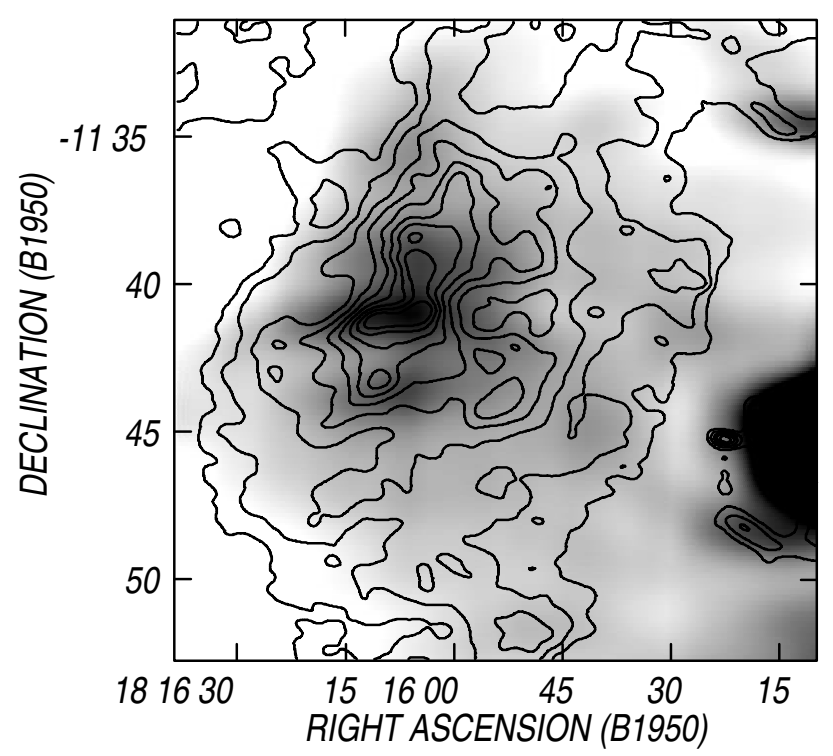

FIG. 6.-Radio continuum contours of G18.8+1.8 superposed on the HIRES infrared image at $60 \mu \mathrm{m}$. The gray scale corresponds to 500 to 1500 $\mathrm{MJy} \mathrm{sr}^{-1}$. The contours are the same as in Fig. 5. 
mates using [S II] favor the densest parts of the nebula, the filling factor could be as low as $f=\left(n_{e} / n_{[S \mathrm{II}}\right)^{2} \simeq 0.005$. We believe that reasonable values for the inner shell as a whole are in the range $f \simeq 0.02-0.15$, which results in electron densities $n_{f} \simeq 180-500 \mathrm{~cm}^{-3}$. The ionized mass in the range $11-$ $29 M_{\odot}$, obtained using the same filling factors, suggests that a nonnegligible fraction of the ionized mass in the inner ring may be stellar ejecta material. The outer ring probably consists of swept-up ISM. Ambient densities $n_{0} \approx 25 \mathrm{~cm}^{-3}$ are obtained by distributing the ionized gas within the volume of a sphere of $2.0 \mathrm{pc}$ in radius.

IR flux densities and dust temperatures have not been estimated for this structure because of the extended background emission and the difficulty of accurately determining the size of the region associated with WR 113. As was done for WR 101 above, we also produced a correlation diagram between $T_{B, 21}$ and $I_{60}$ (Fig. $3 b$ ). Only the emission immediately surrounding WR 113 was considered (in particular, the very strong emission from G18.7+2.0, near the western edge of the VLA image, is not included). The fitted slope is $40.2 \pm 5.7$. The emission originating from WR 113 is clearly thermal. The ratio between the brightness at $60 \mu \mathrm{m}$ and the radio brightness is $R=610 \pm 90$ for this region, thus confirming its thermal nature.

The dust and the ionized gas distributions are consistent with a location of the nebula in the border of the ambient material in the Sh 54 ionized complex. Most of the material to the east and north of the star has probably been blown by the stellar winds during the present W-R phase or previous evolutionary phases. The motion of the star toward the northeast could also be responsible for the present position of the star relative to the nebula. The components of the proper motion of WR 113 listed by the Hipparcos Catalogue $\left(\mu_{\alpha} \cos \delta=3.57 \pm 1.46 \mathrm{mas}_{\mathrm{yr}}^{-1}, \mu_{\delta}=-2.53 \pm 0.91 \mathrm{mas}\right.$ $\left.\mathrm{yr}^{-1}\right)$ and Tycho-2 Catalogue $\left(\mu_{\alpha} \cos \delta=-0.6 \pm 1.4\right.$ mas $\mathrm{yr}^{-1}, \mu_{\delta}=2.2 \pm 1.5 \mathrm{mas} \mathrm{yr}^{-1}$; Hog et al. 2000) are inconclusive since they are so discrepant both in modulus and direction. We note that proper motions for stars at distances greater than $1.5 \mathrm{kpc}$ have large uncertainties.

Finally, a rough estimate of the dynamical age of the ring nebula can be obtained using the expressions by McCray (1983). The dynamical age is thus $t_{6}=0.55 R_{S} / V_{\text {exp }}$, where the constant represents a mean value between the energyand momentum-conserving cases. Adopting $R_{S}=2.3 \mathrm{pc}$ as the radius of the inner ring and $V_{\exp }=5-10 \mathrm{~km} \mathrm{~s}^{-1}$ (since the observed line widths could be explained if the ring nebula is expanding at low velocity; see $\S 1), t_{d} \simeq(1.3-2.5) \times 10^{5}$ yr. The stellar wind luminosity $L_{36}$ necessary to create the nebula, obtained using the expression for the energy-conserving case (Weaver et al 1977) is $L_{36}=0.007-0.05$.

The derived dynamical age suggests that the ring nebula originated during the W-R phase of the star. The observed stellar wind luminosity is $L_{36-\mathrm{obs}}=7.2$, adopting the same values for $\dot{M}$ and $V_{w}$ as in $\S 3.1$. The ratio between the observed and the inferred stellar wind luminosities is $L_{36-\text { obs }} / L_{36}>140$, suggesting that the nebula is in an intermediate stage between the energy- and momentum-conserving cases. This discrepancy between the stellar wind luminosities derived from observations and the energy-conserving model has been reported previously for galactic and extragalactic windblown bubble nebulae (e.g., Nazé et al. 2001). Similar discrepancies were also found in the galactic $\mathrm{H}$ I interstellar bubbles around Of and W-R stars (e.g., Cappa et al. 1999; Cappa \& Herbstmeier 2000).

\section{SUMMARY}

Based on radio continuum observations at $1.465 \mathrm{MHz}$ obtained using the VLA in the DnC array, we have investigated the nature and parameters of the ring nebulae around WR 101 and WR 113. Both nebulae have radio counterparts. G357.5-1.4, associated with WR 101, and G18.8+1.8, related to WR 113, are thermal radio sources. G18.8+1.8 consists of a bright ionized shell and an outer arc barely detectable at radio wavelengths.

The ionized mass and electron density (corrected for filling factor) of G357.5-1.4 are $\approx 230 \pm 40 M_{\odot}$ and $40-55$ $\mathrm{cm}^{-3}$. The high-resolution IRAS images at 60 and $100 \mu \mathrm{m}$ lead to a mass estimate of $\approx 0.3-1.0 M_{\odot}$ and a temperature of $\approx 40 \mathrm{~K}$ for the dust component. The gas-to-dust ratio suggests that the filling factor of the nebula is quite low $(f<0.1)$.

The ionized masses and electron densities in the inner and outer structures in G18.8+1.8 are $\approx 20 \pm 10 M_{\odot}, 180-500$ $\mathrm{cm}^{-3}$ and $\approx 90 M_{\odot}, 40 \mathrm{~cm}^{-3}$, respectively. Again in this case, very low filling factors are inferred.

The electron density in G357.5-1.4 is similar to electron density estimates for galactic W-R ring nebulae classified as $W$ or $R$ (e.g., NGC 2359, RCW 78, G1.2+2.4, and S308; see Esteban et al. 1992; Goss \& Lozinskaya 1995; Cappa et al 1999), while the electron density in the inner shell of G18.8+1.8 is compatible with determinations for E-type and W/E nebulae (e.g., M1-M67, RCW 58, and NGC 6888; Esteban et al. 1992). These results suggest that for G357.5-1.4 and the outer arc of G18.8+1.8 the nebulae consist mainly of swept-up interstellar gas. On the other hand, the inner shell of G18.8+1.8 may contain a relatively large fraction of expelled ejecta material. Studies of chemical abundances are needed to clarify the origin of these nebulae.

The ring nebula around WR 101 and the inner shell associated with WR 113 were probably created during the W-R phase of the stars. The inner shell around WR 113 is probably in an intermediate stage between the energy- and momentum-conserving cases. A kinematic study of the ring nebula associated with WR 101 is necessary to investigate its evolutionary stage. Previous results show that many galactic ring nebulae and $\mathrm{H}$ I interstellar bubbles around $\mathrm{Of}$ and W-R stars are in an intermediate stage between the energy- and momentum-conserving cases (e.g., Cappa et al. 1999; Cappa \& Herbstmeier 2000 and references therein).

$\mathrm{H} \mathrm{I}$ and molecular observations of these nebulae are necessary to investigate the interplay between the ring nebulae and the surrounding material.

We would like to acknowledge the anonymous referee for his or her constructive suggestions. C. C. would like to thank G. Dubner for advice on reducing VLA data. The work of S. P. was supported by the Natural Sciences and Engineering Research Council of Canada and the Fonds FCAR of Québec. The National Radio Astronomy Observatory is a facility of the National Science Foundation operated under cooperative agreement by Associated Universities, Inc. This research was partially supported by Facultad de Ciencias Astronómicas y Geofísicas, Universidad Nacional de La Plata; project 11/G049 (UNLP); and CONICET project PIP 607/98, Argentina. 


\section{REFERENCES}

Broadbent, A., Haslam, C. G. T., \& Osborne, J. L. 1989, MNRAS, 237, 381

Cappa, C. E., Goss, W. M., Niemela, V. S., \& Ostrov, P. 1999, AJ, 118, 948

Marston, A. P. 1991, ApJ, 366, 181

Cappa, C. E., \& Herbstmeier, U. 2000, AJ, 120, 1963

Chu, Y.-H. 1991, in IAU Symp. 143, Wolf-Rayet Stars and Interrelations with Other Massive Stars in Galaxies, ed. K. van der Hucht \& B. Hidayat (Dordrecht: Kluwer), 349

Chu, Y. H., Treffers, R. R., \& Kwitter, K. B. 1983, ApJS, 53, 937

Cichowolski, S., Pineault, S., Arnal, E. M., Testori, J. C., Goss, W. M., \& Cappa, C. E. 2001, AJ, 122, 1938

Condon, J. J., Cotton, W. D., Greisen, E. W., Yin, Q. F., Perley, R. A., Taylor, G. B., \& Broderick, C. K. 1998, AJ, 115, 1693

Conti, P. S., \& Vacca, W. D. 1990, AJ, 100, 431

Draine, B. T., \& Lee, H. M. 1984, ApJ, 285, 89

Eenens, P. R. J., \& Williams, P. M. 1994, MNRAS, 269, 1082

Esteban, C., \& Rosado, M. 1995, A\&A, 304, 491

Esteban, C., Vilchez, J. M., Smith, L. J., \& Clegg, R. E. S. 1992, A\&A, 259 629

Felli, M., \& Perinotto, M. 1979, A\&A, 76, 69

Fich, M., \& Terebey, S. 1996, ApJ, 472, 624

Fürst, E., Reich, W., \& Sofue, Y. 1987, A\&AS, 71, 63

Gervais, N., \& St-Louis, N. 1999, AJ, 118, 2394

Gonzalez, J., \& Rosado, M. 1984, A\&A, 134, L21

Goss, W. M., \& Day, G. A. 1970, Australian J. Phys., Astrophys. Suppl., 13,3

Goss, W. M. \& Lozinskaya, T. A. 1995, ApJ, 439, 637

Goss, W. M., \& Shaver, P. A. 1970, Australian J. Phys. Astrophys. Suppl., 14,1

Haynes, R. F., Caswell, J. L., \& Simons, L. W. J. 1978, Australian J. Phys. Astrophys. Suppl., 45, 1

Hog, E., et al. 2000, A\&A, 355, L27

Israel, F. P., \& Felli, M. 1976, A\&A, 50, 47

Johnson, H. M., \& Hogg, D. E. 1965, ApJ, 142, 1033

Koesterke, L., \& Hamann, W.-R. 1995, A\&A, 299, 503

Kothes, R., \& Kerton, C. 2002, A\&A, submitted

Lamontagne, R., Moffat, A. F. J., Drissen, L., Robert, C., \& Matthews, J. M. 1996, AJ, 112, 2227

Leitherer, C., Chapman, J. M., \& Koribalski, B. 1997, ApJ, 481, 898

Lockman, F. J. 1989, ApJS, 71, 469

- $1995 \mathrm{a}, \mathrm{AJ}, 109,1839$

. 1995b, AJ, 109, 2257

1996, AJ, 112, 2828

. 1997, ApJ, 475, 188

Marston, A. P., Chu, Y.-H., \& Garcia-Segura, G. 1994a, ApJS, 93, 229

Marston, A. P., Yocum, D. R., Garcia-Segura, G., \& Chu, Y.-H. 1994b, ApJS, 95, 151

Mathis, J. S., Cassinelli, J. P., van der Hucht, K. A., Prusti, T., Wesselius, P. R., \& Williams, P. M. 1992, ApJ, 384, 197

McCray, R. 1983, Highlights Astron., 6, 565

Mezger, P. G., \& Henderson, A. P. 1967, ApJ, 147, 471

Miller, G., \& Chu, Y.-H. 1993, ApJS, 85, 137

Moon, D. S., \& Koo, B. C. 1994, J. Korean Astron. Soc., 27, 81

Nazé, Y., Chu, Y.-H., Points, S. D., Danforth, C. W., Rosado, M., \& Rosie Chen, C.-H. 2001, AJ, 122, 921

Nugis, T., Crowther, P. A., \& Willis, A. J. 1998, A\&A, 333, 956

Nugis, T., \& Lamers, H. J. G. L. M. 2000, A\&A, 360, 227

Osterloh, M., Henning, Th., \& Launhardt, R. 1997, ApJS, 110, 71

Pineault, S., Landecker, T. L., Madore, B., \& Gaumont-Gay, S. 1993, AJ, 105,1060

Reich, W., Fürst, E., Reich, P., \& Reif, K. 1990, A\&AS, 85, 633

Sharpless, S. 1959, ApJS, 4, 257

Shaver, P. A., \& Goss, W. M. 1970a, Australian J. Phys. Astrophys. Suppl., 14,77

1970b, Australian J. Phys. Astrophys. Suppl., 14, 133

Smith, L. S., Shara, M. M., \& Moffat, A. F. J. 1990, ApJ, 358, 229

Van Buren, D., \& McCray, R. 1988, ApJ, 329, L93

van der Hucht, K. A. 2001, NewA Rev., 45, 135

Veen, P. M., van Genderen, A. M., van der Hucht, K. A., Li, A., Sterken, C., \& Dopminik, C. 1998, A\&A, 329, 199

Weaver, R., McCray, R., Castor, J., Shapiro, P., \& Moore, R. 1977, ApJ, 218, 377

Wendker, M. J., Smith, L. F., Israel, F. P., Habing, H. J., \& Dickel, H. R. 1975, A\&A, 42, 173

Williams, P. M. 1995, in IAU Symp. 163, Wolf-Rayet Stars: Binaries, Colliding Winds, Evolution, ed. K. A. van der Hucht, P. M. Williams, (Dordrecht: Kluwer), 335

Williams, P. M., van der Hucht, K. A., \& The, P. S. 1987, A\&A, 182, 91 